\title{
Enhancing theory of mind in behavioural variant frontotemporal dementia with transcranial direct current stimulation
}

\author{
Maria Cotelli ${ }^{1}$ - Mauro Adenzato ${ }^{2,3}$ (D) Valentina Cantoni ${ }^{4,5} \cdot$ Rosa Manenti $^{1} \cdot$ Antonella Alberici $^{4} \cdot$ Ivan Enrici $^{3,6}$. \\ Alberto Benussi $^{5}$ - Valentina Dell'Era ${ }^{5}$. Elisa Bonetta ${ }^{5}$ - Alessandro Padovani ${ }^{5}$ - Barbara Borroni ${ }^{5}$
}

Published online: 11 July 2018

(C) Psychonomic Society, Inc. 2018

\begin{abstract}
Behavioural variant frontotemporal dementia (bvFTD) is a form of frontotemporal degeneration characterized by early changes in personality, emotional blunting, and/or loss of empathy. Recent research has highlighted that these features may be at least partially explained by impairments in the theory of mind (ToM; i.e., the ability to understand and predict other people's behaviour by attributing independent mental states to them). The aim of this randomized, double-blind, placebo-controlled study was to test the hypothesis that transcranial direct current stimulation (tDCS) over the medial frontal cortex (MFC) selectively enhances communicative intention processing, a specific ToM ability. Using a single-session online design, we administered a ToM task that measures the ability to represent other people's private and communicative intentions during active or sham tDCS to 16 bvFTD patients. To assess the impact of dementia on performance on the ToM task, we included 16 age-matched healthy volunteers who were asked to perform the entire experimental ToM task. BvFTD is characterized by an impairment in the comprehension of both communicative and private intentions relative to a healthy control group and by a disproportional impairment in communicative intention compared with private intention processing. Significant and selective accuracy improvement in the comprehension of communicative intentions after active stimulation was observed in patients with bvFTD. This is the first study that analyses ToM ability in patients with bvFTD using tDCS stimulation. Our findings could potentially contribute to the development of an effective, noninvasive brain stimulation treatment of ToM impairments in patients with bvFTD.
\end{abstract}

Keywords Anodal transcranial direct current stimulation (tDCS) · Behavioural variant frontotemporal dementia (bvFTD) $\cdot$ Theory of mind (ToM) $\cdot$ Medial frontal cortex (MFC)

Maria Cotelli and Mauro Adenzato contributed equally to this work.

Mauro Adenzato

mauro.adenzato@unito.it

1 Neuropsychology Unit, IRCCS Centro San Giovanni di DioFatebenefratelli, Brescia, Italy

2 Department of Psychology, University of Turin, via Po 14, 10123 Turin, Italy

3 Neuroscience Institute of Turin, Turin, Italy

4 Department of Neuroscience, Psychology, Drug Research and Child Health, University of Florence, Florence, Italy

5 Centre for Neurodegenerative Disorders, Neurology Unit, Department of Clinical and Experimental Sciences, University of Brescia, Brescia, Italy

6 Department of Philosophy and Educational Sciences, University of Turin, Turin, Italy
Behavioural variant frontotemporal dementia (bvFTD) is a form of frontotemporal degeneration characterized by early and progressive changes in personality, emotional blunting, and/or loss of empathy (Rascovsky et al., 2011). In bvFTD, difficulty in modulating behaviour results in socially inappropriate responses or activities, such as social misconduct, sexual disinhibition, and/or financial loss, which results in a poorer prognosis, faster institutionalization and a higher level of distress for caregivers (Rascovsky et al., 2011).

Recent research has highlighted that the personality changes and the breakdown in social conduct frequently seen in bvFTD may be explained by an impairment in the ability to explain and predict other people's behaviour by attributing independent mental states to them, which constitutes the core postulates of the theory of mind (ToM; Adenzato \& Poletti, 2013; Poletti, Enrici, \& Adenzato, 2012). ToM abilities form the basis of any social interaction, and they have been proposed to rely on a distributed 
neural network including the complex constituted by the right and left temporoparietal junctions, the temporal poles, the precuneus, and the medial frontal cortex (MFC; Carrington \& Bailey, 2009; Kampe, Frith, \& Frith, 2003). In particular, studies that analysed the neural correlates of intention processing found that the MFC and the temporoparietal junctions are differentially engaged depending on the nature of the intention involved, with a progressive recruitment of the ToM network along the theoretical distinction of the private versus social/ communicative dimension (Ciaramidaro et al., 2007; Enrici, Adenzato, Cappa, Bara, \& Tettamanti, 2011; Tettamanti et al., 2017; Walter et al., 2004). Recent studies have used ToM tasks to investigate ToM deficits in bvFTD, in which a significant impairment in these abilities was observed (Bertoux \& Hornberger, 2015; Brioschi Guevara et al., 2015; Eslinger et al., 2007; Gregory et al., 2002; Henry, Phillips, \& von Hippel, 2014; Ibanez \& Manes, 2012; Le Bouc et al., 2012; Lough et al., 2006; Pardini et al., 2013; Pardini et al., 2015; Snowden et al., 2003; Torralva et al., 2007).

Currently, there is growing interest in transcranial direct current stimulation (tDCS), a noninvasive technique purported to modulate neural activity via weak, externally applied electric fields. tDCS generates an increase or a decrease in neuronal excitability that can modulate cognitive task performance by applying weak electrical currents directly to the head over a long period of time, usually on the order of minutes. tDCS delivers a weak, polarizing electrical current to the cortex through a pair of electrodes, and brain excitability can be increased via anodal stimulation or decreased via cathodal stimulation depending on the polarity of the current flow (Nitsche et al., 2008; Paulus, 2011; Polania, Nitsche, \& Ruff, 2018). Using this technique, short-term facilitation effects on the cognitive functions of normal subjects and neurodegenerative and psychiatric patients have been previously reported (Brunoni et al., 2012; Martins, Fregni, Simis, \& Almeida, 2017; Vallar \& Bolognini, 2011). It has also been shown that a single tDCS session can influence performance on a social cognition task in healthy subjects (Adenzato et al., 2017; Conson et al., 2015; Fumagalli et al., 2010; Mai et al., 2016; Martin, Dzafic, Ramdave, \& Meinzer, 2017; Martin, Huang, Hunold, \& Meinzer, 2017; Santiesteban, Banissy, Catmur, \& Bird, 2012).

Application of tDCS (anode over the left frontal cortex) has already been shown to improve language deficits in patients with FTD (Cotelli et al., 2016; Cotelli et al., 2014; Gervits et al., 2016), but to the best of our knowledge, no study has investigated the capacity of tDCS to ameliorate ToM performance in these patients.

In the current study, we applied anodal tDCS over the MFC (Fpz site, with the cathode between $\mathrm{Oz}$ and Inion) to modulate
ToM performance in bvFTD patients. We used an adapted version of a ToM task, measuring the ability to represent other people's private and communicative intentions from the observation of their daily actions. The theoretical distinction between private and communicative intentions has been proposed within the intention processing network (IPN) model (Bara, Enrici, \& Adenzato, 2016; Ciaramidaro et al., 2007). According to this model, private intentions only involve the actor satisfying a particular goal (e.g., working in the kitchen to prepare oneself a meal). Conversely, in communicative intentions, the goal of the actor is satisfied only if at least one other person is involved (e.g., pointing to a bottle to request it). Thus, while a private intention can be realized by the actions of an isolated person, a communicative intention can occur only during social interaction. The IPN model has been supported by experimental studies showing that a set of brain areas, i.e., the right and left temporoparietal junctions, precuneus, and MFC, are differentially involved in comprehending these different types of intentions (Bara, Ciaramidaro, Walter, \& Adenzato, 2011; Ciaramidaro et al., 2007; Walter et al., 2004; Walter et al., 2009). Strong empirical evidence shows that the MFC is engaged during social inferences, in particular, during social scripts that do not concern only a single actor but that describe adequate social actions for all of the actors involved in a social context (Van Overwalle, 2009, 2011). Moreover, meta-analysis studies have implicated the precuneus in the elaboration of contextual information and identification of situational structure, whereas the temporoparietal junctions were generally associated with the identification of end-state behaviours (Van Overwalle, 2009; Van Overwalle \& Baetens, 2009). In particular, according to Van Overwalle (2009), the temporoparietal junctions, along with the precuneus and MFC, are involved in the broader process of goal identification in a social context.

The rationale of the present study stemmed from evidence showing that the MFC is already affected in the early stages of bvFTD (Borroni et al., 2007; Buhour et al., 2017; Pan et al., 2012; Salmon et al., 2003; Schroeter, Raczka, Neumann, \& von Cramon, 2008), and that the MFC is involved in the comprehension of communicative intentions rather than in the comprehension of private intentions (Bara et al., 2011; Ciaramidaro et al., 2007; Enrici et al., 2011; Tettamanti et al., 2017; Walter et al., 2004).

In consideration of the bvFTD neuropsychological profile previously described, here, we hypothesized that in the early stages of bvFTD, (a) the comprehension of communicative and private intentions are impaired compared with a healthy control (HC) group; (b) the comprehension of communicative intentions is more impaired than the comprehension of private intentions as a consequence of the role played by the MFC in communicative intentions, and (c) anodal tDCS over the MFC (Fpz site, with the cathode between $\mathrm{Oz}$ and Inion) is able to enhance communicative intention performance. 


\section{Material and methods}

\section{Patients and control groups}

Patients fulfilling current clinical criteria for probable bvFTD (Rascovsky et al., 2011) were recruited at the Centre for Neurodegenerative Disorders, Neurology Unit, University of Brescia, Italy.

At enrolment, patients underwent an extensive clinical and neurological evaluation and a standardized neuropsychological assessment. Diagnosis was corroborated by the presence of frontotemporal atrophy identified with conventional structural imaging (Borroni et al., 2015). Genetic screening of the most frequent monogenic causes of FTD in Italy was carried out, and Granulin (GRN) mutations and C9orf72 gene expansion were considered (Premi et al., 2017).

Neuropsychological testing included the Frontotemporal Lobar Degeneration-Clinical Dementia Rating (FTLD-CDR) scale, the Mini-Mental State Examination, verbal fluency (phonemic and semantic cue), the Rey-Osterrieth complex figure copy and recall, the story recall test, the digit span, the Trail Making test (Parts A and B) and the Token test (Lezak, Howieson, \& Loring, 2012). Behavioural disturbances were evaluated with the Frontal Behavioural Inventory (FBI).

Patients with severe behavioural disorders, or FTLDCDR $>10$, were excluded to prevent any confounding in the experimental task results. Moreover, patients with contraindications to performing tDCS, such as a history of seizures, major head trauma, past brain surgery, a brain metal implant or a pacemaker, were excluded as well.

To assess the impact of dementia on ToM tasks, we also included 16 healthy volunteers, matched for age and education $(N=16$, four males, age $=67.7 \pm 7$ years, education $=10.5 \pm 4$ years). HC participants were asked to perform the entire experimental ToM task without tDCS in order to compare their comprehension of communicative and private intentions to that of bvFTD patients. HC participants with no history of mental illness or cognitive decline, no motor or cognitive complaints, normal objective cognitive performance in all the administered neuropsychological tests, normal scores in functional assessment, and absence of mood and anxiety disorders were included.

The Brescia Hospital Ethics Committee approved the study (NP2388), and all participants provided written informed consent according to the Declaration of Helsinki.

\section{Study design}

The present work was a randomized, double-blind, shamcontrolled study. Each patient received both active and sham tDCS over the MFC (Fpz site, with the cathode between Oz and Inion) in randomized order in two different sessions, separated by at least 2 weeks; the first session was assigned in a 1:1 ratio of active or sham tDCS. The patient and the examiner who performed ratings were blind to the type of tDCS delivered. During each session, a video version of the ToM task was administered, and video-clips were presented in a randomized order (see below). According to data from the literature, the changes in excitability induced by a single session of anodal tDCS are expected to last for up to $1 \mathrm{hr}$ if the stimulation is sufficiently long (Nitsche et al., 2007; Nitsche et al., 2003; Nitsche \& Paulus, 2000). Hence, patients were expected to return to their initial clinical status between the two sessions of stimulation.

\section{Theory of mind tasks}

All of the participants performed the attribution of intentions (AI) task to test the effect of tDCS on cognitive ToM ability. The AI task is a video version of a cognitive ToM task previously used in young individuals (Adenzato et al., 2017). During the AI task, participants were asked to demonstrate their comprehension of the displayed stories (short videos of 1,500 milliseconds-development phase) by choosing the most appropriate story ending (out of two concluding pictures displayed until the responseresponse phase). The two possible story endings were shown simultaneously on the left and on the right side of the screen until the participant responded by pressing the corresponding button (right for the right figure and left for the left figure) on the button box as quickly as possible; the correct picture represented a probable conclusion, whereas the incorrect picture represented an improbable ending (see Fig. 1).

Two types of experimental conditions were included in the task and were presented in the video-clips: (a) private intention condition (PInt), in which participants were required to recognize another person's intention while watching his or her isolated actions (e.g., hanging a picture on the wall), and (b) communicative intention condition (CInt), in which participants were required to recognize another person's communicative intention during a social interaction (e.g., asking another person to obtain a glass of water for them).

The present study included 34 video stories for each condition, for a total of 68 stories.

Participants were seated in a quiet room facing a computer monitor placed $60 \mathrm{~cm}$ away from them. The stimuli were presented using Presentation software (Version 16.3, www.neurobs.com) running on a personal computer with a 15 -inch screen. Visual location (right and left side of the screen) of the correct answer was randomized. AI accuracy and reaction times (RTs) were recorded.

The items were divided into two blocks (17 PInt and 17 CInt stimuli each) corresponding to the two types of stimulation (active and sham stimulation). The visual complexity of the scenes was matched and counterbalanced between the two experimental sessions. Moreover, four additional stimuli were selected to be used in a training session (two PInt and two CInt stimuli). Each experimental block took approximately 8 minutes to complete. 
a

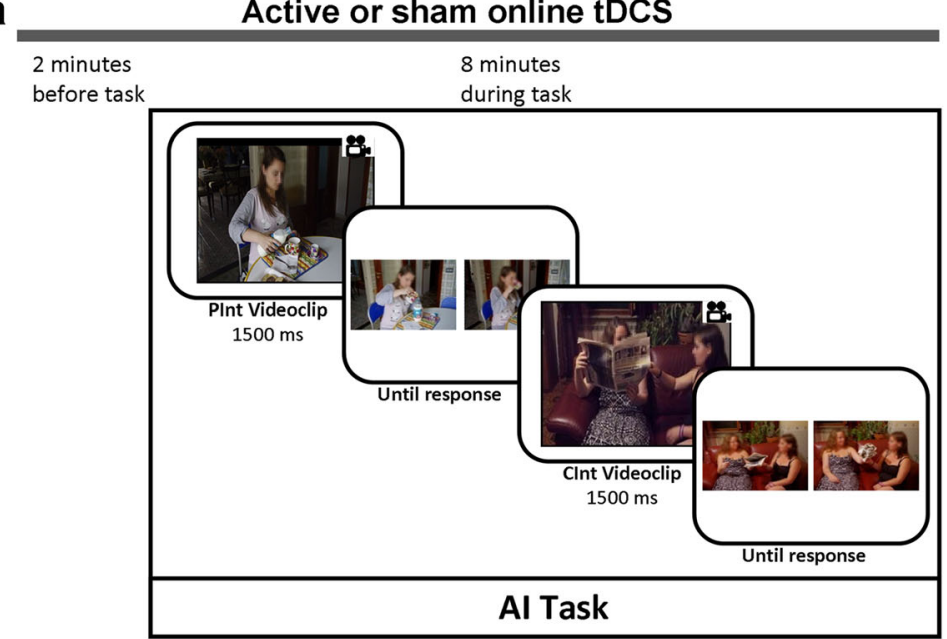

b

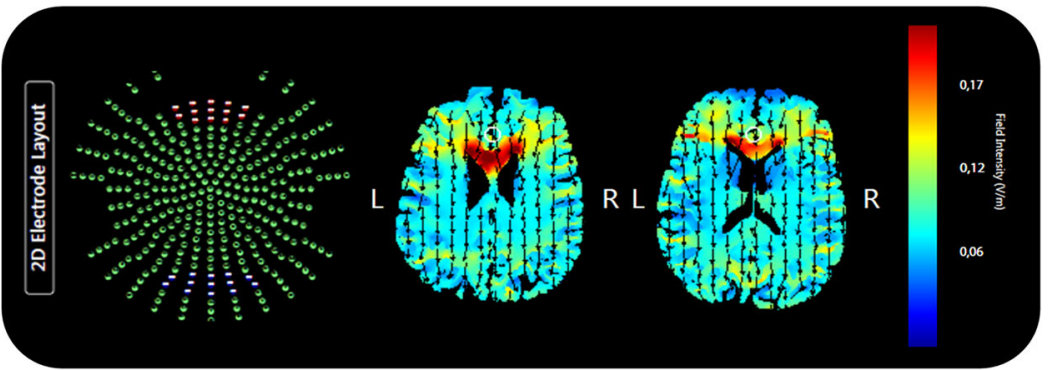

Fig. 1 a Experimental design. Active or sham tDCS was applied 2 minutes before the beginning of the experimental block and covered the entire AI task. In the AI task, a short video was played, and the participant was asked to choose the picture representing a logical story ending by pushing one of the two buttons on the button box. One example of each stimulus condition (CInt and a PInt) is displayed (see text for details). b

\section{The tDCS procedure}

In the present study transcranial direct current stimulation (tDCS) was applied using a battery-driven, constant-current stimulator (HDCstim, Newronika, Milan, Italy) through a pair of saline-soaked sponge electrodes $(7 \mathrm{~cm} \times 5 \mathrm{~cm})$.

The target area for tDCS was the MFC, recognized as pivotal for intention processing in previous studies (reference Montreal National Institute [MNI] coordinates: 0, 60, 18; see Ciaramidaro et al., 2007; Poreisz, Boros, Antal, \& Paulus, 2007; Walter et al., 2004; Walter et al., 2009). Accordingly, the anode was placed over Fpz and the cathode was placed between Inion and $\mathrm{Oz}$ (see Fig. 1b), according to the 10-20 electroencephalography (EEG) international system (Herwig, Satrapi, \& Schonfeldt-Lecuona, 2003). Specifically, an EEG cap was gently secured on the head of each subject and positioned with $\mathrm{Cz}$ at the vertex, and $\mathrm{FPz}$ was marked with a pencil and identified as the centre of the anode. The EEG cap was then removed to allow tDCS montage. During active tDCS, a constant current of $1.5 \mathrm{~mA}$ was applied for 10 minutes (a ramping period of 10 seconds at the beginning of the stimulation), starting 2 minutes before the beginning of the task and finishing after the end of the task. The current density
Current flow model of tDCS montage. Anode over MFC and cathode between Inion and $\mathrm{Oz}$, using two $7 \times 5$ sponge pads represented in transverse views from the Male 1 model in the Soterix HD Targets software (Soterix Medical). Black arrows represent the direction of current flow. (Colour figure online)

$\left(0.043 \mathrm{~mA} / \mathrm{cm}^{2}\right)$ was maintained below the safety limits (Poreisz et al., 2007). During sham stimulation, the tDCS montage was the same, but the current was turned off 10 seconds after the stimulation began, making this condition indistinguishable from the active stimulation.

At the end of each session, patients filled out a questionnaire in order to exclude perceptual sensation differences between active and sham stimulation, as well as, side effects. The order of the two stimulation conditions (sham and active) was randomized across participants (i.e., $50 \%$ of participants received active stimulation on Session 1 and sham stimulation on Session 2) and was executed at the same day and time to minimize the likelihood of confounding interference effects.

\section{Statistical analyses}

Statistical analyses were performed using Statistica software (version 10; www.statsoft.com).

Considering that the data were normally distributed (RTs: Kolmogorov-Smirnov test: $d=0.19, p=.20$; Accuracy: Kolmogorov-Smirnov test: $d=0.16, p>.30$ ), AI task performance (accuracy and RT) recorded in the bvFTD group in the sham tDCS condition was compared with performance 
obtained in the AI task by an age, gender, and education matched $\mathrm{HC}$ group, using an analysis of variance (ANOVA), including the two types of stimuli (PInt and CInt) as withinparticipants factors, and the group (bvFTD and HC) as between-participants factors.

Moreover, considering that the data were normally distributed (RTs: Kolmogorov-Smirnov test: $d=0.14, p=.20$; Accuracy: Kolmogorov-Smirnov test: $d=0.17, p=.10$ ), AI task performance (accuracy and RT) was analysed using repeated-measures analysis of covariance (ANCOVA), including the two types of stimulation (anodal or sham) and the two types of stimuli (PInt and CInt) as withinparticipants factors, and the FTLD-CDR score as a covariate. Post hoc analysis was carried out using planned comparisons contrasting the two stimulation conditions in each stimulus task. Sensation scores were compared between anodal tDCS and sham tDCS using a Wilcoxon matched-pair test. Statistical significance was set at $p<.05$. Statistical power and effect size (Cohen's $d$ ) analyses were estimated using G*Power 3.1 (Faul, Erdfelder, Lang, \& Buchner, 2007).

\section{Results}

We included 20 consecutive patients with mild bvFTD according to the inclusion criteria; three patients were then excluded for disease severity and one patient was excluded due to a history of seizures. Thus, the present analysis was conducted on 16 bvFTD patients. Demographic and clinical characteristics of the included subjects are reported in Table 1.

Three out of the 16 patients had monogenic FTD $(n=2$ GRN Thr272fs, and $n=1$ C9orf72) expansion.

\section{Behavioural performance in bvFTD patients and in HC on attribution of intentions (AI) task}

Reaction-time analysis showed a significant effect of group, $F(1,30)=34.49, p=.000002, \eta^{2}=0.54,1-\beta=0.99$, suggesting that the bvFTD group was slower than the HC group (HC: $1887.5 \mathrm{~ms} \pm 391$; bvFTD: $3631.3 \mathrm{~ms} \pm 980$ ) in both CInt and PInt conditions. No other effect reached statistical significance.

Accuracy analysis indicated a significant effect of group, $F(1,30)=20.13, p=.0001, \eta^{2}=0.40,1-\beta=0.99$, and of the type of stimulus, $F(1,30)=10.72, p=.003, \eta^{2}=0.26,1-\beta=$ 0.99 . These results suggest that the accuracy recorded in the CInt condition was significantly worse than the accuracy in the PInt condition in both groups (CInt: $80.9 \% \pm 17$; PInt: $87.5 \% \pm 13$ ) but that the bvFTD group was less accurate than the HC group (HC: $91.2 \% \pm 7$; bvFTD: $75.2 \% \pm 14$ ). See Fig. 2 for details.

\section{Effects of tDCS on Al in bvFTD}

Reaction-time analysis showed no significant effect for type of stimulation, $\mathrm{F}(1,14)=0.32, p=.58, \eta^{2}=0.02,1-\beta=$ 0.18 ; , type of stimulus, $F(1,14)=2.36, p=.15, \eta^{2}=0.14,1-$ $\beta=0.65$; and interaction between factors, $F(1,14)=0.01, p=$ $.94, \eta^{2}<0.01,1-\beta=0.06$; CInt condition: $3647 \pm 282$ [sham tDCS], $4232 \pm 513$ [active tDCS]; PInt condition: $3614 \pm 234$ [sham tDCS], $4213 \pm 567$ [active tDCS]).

Accuracy analysis indicated a significant effect of the interaction between type of stimulus (CInt vs. PInt), type of stimulation (active vs. sham), and FTLD-CDR, $F(1,14)=$ $4.99, p=.042, \eta^{2}=0.26,1-\beta=0.99$. No other effect reached statistical significance.

Accuracy recorded in the CInt condition was significantly worse than accuracy in the PInt condition during sham tDCS $(p=.024)$, suggesting an impairment of CInt in early bvFTD (see Fig. 3). Moreover, post hoc analysis showed an increase in accuracy induced by active tDCS compared with sham tDCS in the CInt condition (77.2 \pm 15 [active tDCS] vs. 70.6 \pm 17 [sham tDCS]; $p=.048$ ), whereas no such effect was observed in the PInt condition (77.6 \pm 16 [active tDCS] $v s$. $79.8 \pm 15$ [sham tDCS]; $p=.55$; see Fig. 3).

\section{Sensations questionnaire}

The sensations questionnaire completed by the bvFTD patients at the end of each stimulation session showed that all of them tolerated the stimulation well. The Wilcoxon matched-pairs test showed that perceptual sensations reported after active and sham stimulation sessions were not significantly different $(T=11.0, z=1.68 ; \mathrm{p}=.09)$. Accordingly, there is no reason to reject the blinded nature of this study.

\section{Discussion}

In recent years, there has been a resurgence of interest in ToM, particularly in its fundamental role in many social and communicative interactions (Adenzato \& Garbarini, 2006; Bara et al., 2016; Di Tella et al., 2015; Enrici et al., 2011; Enrici et al., 2015; Tettamanti et al., 2017). This is the first study that analyses ToM ability in patients with bvFTD using tDCS stimulation. The aim of the present study was to assess for the presence of an impairment in communicative and private intentions in the mild stage of bvFTD. In particular, the prediction was that a more severe impairment would be present for communicative intentions in patients with bvFTD as a consequence of the role played by the MFC in communicative intentions (Bara et al., 2011; Ciaramidaro et al., 2007; Enrici et al., 2011; Tettamanti et al., 2017; Walter et al., 2004). Additionally, another aim of the present study was to investigate whether the application of anodal tDCS over the MFC 
Table 1 Demographic and clinical characteristics of patients with behavioural variant frontotemporal dementia $(n=16)$

\section{Demographic and clinical features}

Age (years)

$64.9(8.6)$

Gender (male/female)

$13 / 3$

Education (years)

$9.4(5.0)$

$1.3(1.7)$

5.9 (3.9)

\section{FTLD-CDR}

Neuropsychological assessment

Screening for dementia

Mini-Mental State Examination (MMSE)

Praxia

Rey-Osterrieth complex figure: Copy

\section{Memory}

Rey-Osterrieth complex figure: Recall

Story recall

Digit span

Raw score

$25.0(4.0)$

$27.0(5.5)$

$8.8(7.0)$

$9.9(5.5)$

$5.0(1.0)$

Attentional functions

Trail Making Test, A

Trail Making Test, B

$89.2(74.4)$

$189.1(167.5)$

Language

Fluency: Phonemic

$24.6(11.7)$

Fluency: Semantic

Token Test

30.9 (1.5)

$16.6(10.5)$

\section{Behavioural disturbances}

Frontal Behavioural Inventory (FBI)
Adjusted score

23.3 (3.6)

$28.0(5.0)$

$9.5(7.1)$

$11.7(5.8)$

$5.1(0.9)$

$73.2(72.1)$

151.9 (151.4)

$27.2(10.5)$

$34.9(8.7)$

30.3 (1.5)
* Cut-off

$\geq 24$

$>9.46$

$>7.5$

$>4.25$

$<94$

$<283$

$>16$

$>24$

$>26.25$

*Cut-off scores according to Italian normative data are reported. Values are mean, standard deviations between brackets. Bold data indicate scores below the cut-off. FTLD-CDR = FTLD-modified Clinical Dementia Rating scale; bvFTD = behavioural variant frontotemporal dementia

a

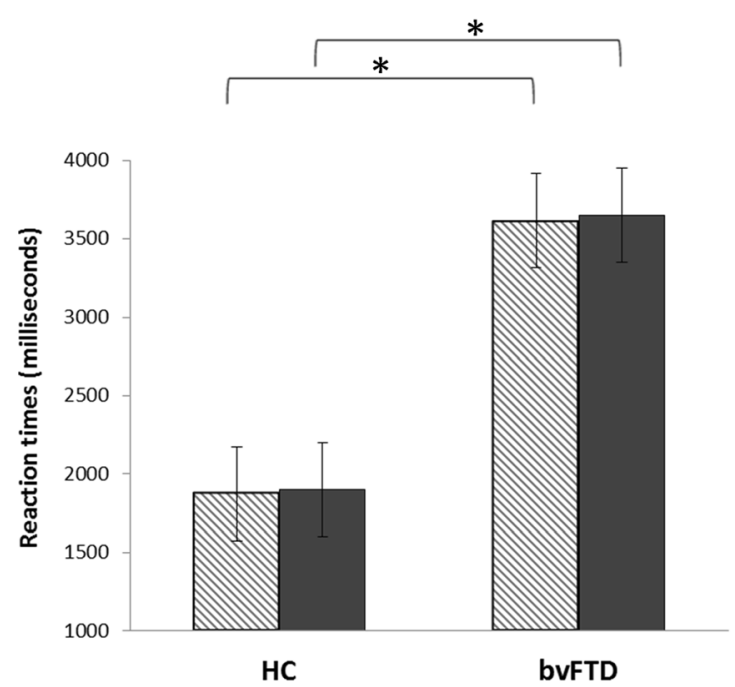

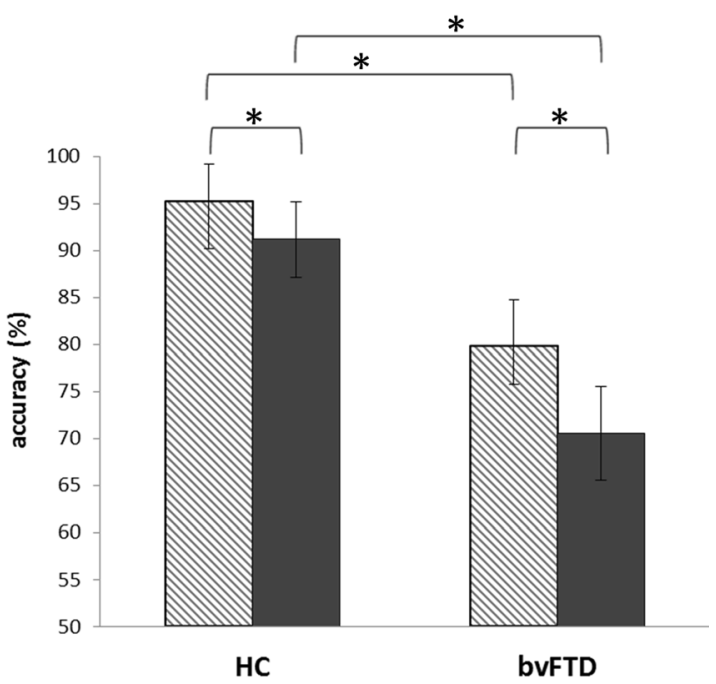

PInt

Fig. 2 Reaction times (a) and accuracy (b) recorded in the attribution of intentions (AI) task (CInt and PInt conditions) in behavioural variant frontotemporal dementia (bvFTD) and healthy control (HC) plotted separately. bvFTD patients' accuracy and reaction times recorded in the CInt b

Accuracy

HC

bvFTD and PInt conditions were significantly worse than those of the HC group. Accuracy was significantly worse on the CInt task than on the PInt task. Asterisks indicate significant effects $(p<.05)$ 


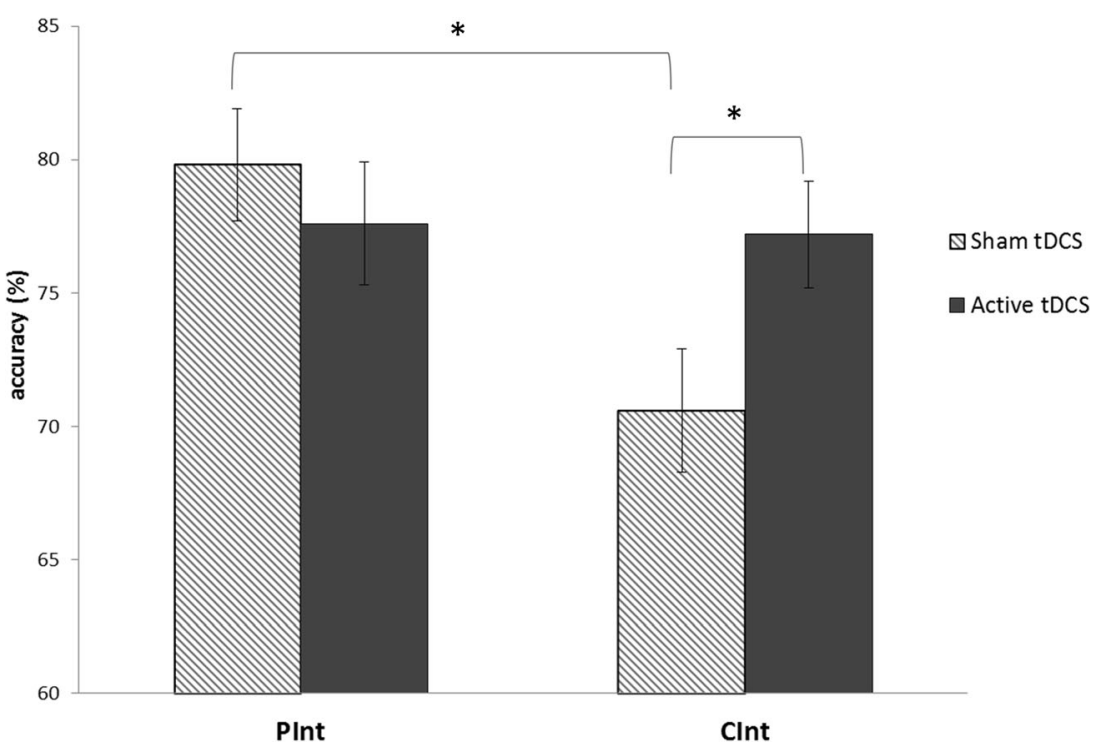

Fig. 3 Transcranial direct current stimulation (tDCS) effects on accuracy in the attribution of intentions task in behavioural variant frontotemporal dementia for active tDCS and sham tDCS (CInt and PInt conditions plotted separately)separately). The accuracy of bvFTD patients recorded in the CInt condition was significantly worse than the accuracy recorded

in the PInt condition during sham tDCS. Furthermore, accuracy on the AI task improved after active anodal tDCS over the MFC (Fpz site, with the cathode between $\mathrm{Oz}$ and Inion) compared with sham stimulation selectively for the CInt stimuli. Asterisks indicate significant effects $(p<.05)$

(Fpz site, with the cathode between $\mathrm{Oz}$ and Inion) selectively enhances communicative intention processing in these patients. The results of the present study revealed that mild bvFTD is characterized by an impairment in the comprehension of private and communicative intentions compared with $\mathrm{HC}$, with a disproportional impairment in the comprehension of communicative intentions.

Very few studies have applied tDCS to patients with FTD, and almost all have mainly focused on the treatment of language deficits in patients with primary progressive aphasia in clinical settings (Cotelli et al., 2016; Cotelli et al., 2014; Gervits et al., 2016; Roncero et al., 2017). An improvement in naming ability was found by Cotelli et al. (2014) after applying anodal tDCS to the left dorsolateral prefrontal cortex. They found that tDCS stimulation facilitated lexical retrieval processes in these patients. A general improvement in linguistic performance in the domains of speech production and grammatical comprehension was found by Gervits et al. (2016) after two weeks of daily tDCS. Anodal tDCS stimulation of the left frontotemporal region (with the cathode over the left occipitoparietal region) led to significant improvements on a variety of linguistic measures that were sustained for at least 3 months following tDCS. Recently, Roncero et al. (2017) applied tDCS to the inferior parietal lobe during picture-naming training on a mixed group of patients with Alzheimer's disease and progressive aphasia and found significant improvement in picture naming compared with placebo.

To the best of our knowledge, no prior studies have applied tDCS to patients with bvFTD. The only exception is a single case study in a clinical setting of a 45-year-old woman with bvFTD (Agarwal et al., 2016). The authors applied anodal

tDCS stimulation to the left dorsolateral prefrontal cortex (with the cathode over the right supraorbital region) for 10 sessions over 5 days. They found significant improvement from the third day of tDCS onward in behavioural disturbances and socio-occupational functioning, in particular in executive and planning skills. Moreover, the patient maintained improvement during follow-up visits over the next 7 months.

Our findings corroborate previous studies of marked impairment in the domain of social cognition in patients with bvFTD (Adenzato, Cavallo, \& Enrici, 2010; Baez et al., 2017; Cavallo, Enrici, \& Adenzato, 2011; Dermody et al., 2016; Eslinger et al., 2007; Gregory et al., 2002; Irish, Hodges, \& Piguet, 2014; Kipps \& Hodges, 2006; Lough, Gregory, \& Hodges, 2001; Lough \& Hodges, 2002; Lough et al., 2006; Snowden et al., 2003; Torralva et al., 2007) and suggest that communicative intentions are disproportionately affected in bvFTD.

Furthermore, our data show that tDCS over the MFC is able to selectively increase the accuracy of comprehension of communicative intentions.

These observations might have important implications in clinical settings for both patients and caregivers, as social cognition impairment represents one of the most distressful symptoms of bvFTD, implying a worse disease progression, faster institutionalization, higher use of off-label medications, and caregiver burden (Brune \& Brune-Cohrs, 2006; Pezzati et al., 2014). The evidence that ToM performance might be improved by tDCS provides a potential intervention approach for 
this disease, still orphan of any pharmacological treatment (Boxer \& Boeve, 2007; Perry \& Miller, 2001).

In discussing our results, the proposed link between the progressive degeneration of the medial frontal structures characterizing the early stages of the bvFTD and the ToM deficits in these patients is of particular relevance (Adenzato et al., 2010; Adenzato \& Poletti, 2013). This observation is at the core of our hypothesis of a selectively enhanced performance in the comprehension of communicative intentions after active stimulation of the MFC, which in turn enhances cortical excitability. In fact, in a set of previous studies (Ciaramidaro et al., 2007; Walter et al., 2004; Walter et al., 2009), it has been demonstrated that the right temporoparietal junction is sufficient for the comprehension of mental states of other individuals, as long as those individuals are acting outside the context of social interaction (the PInt experimental condition of this study), whereas the MFC is necessary for the comprehension of the intentions of others who are specifically involved in communicative interactions (our CInt experimental condition; Ciaramidaro et al., 2007; Walter et al., 2004; Walter et al., 2009).

Accordingly, the specific role of the MFC in the CInt rather than in the PInt condition is confirmed by patient performance in the sham condition, in which a statistically significant difference was found between the two ToM tasks in bvFTD patients, with better performance in the PInt condition. Furthermore, active tDCS over the MFC was shown to selectively improve CInt processing.

However, we acknowledge that this study has some limitations. A control brain area should be taken into consideration in future studies, to further confirm the specificity of the present results, and the inclusion of patients with other neurodegenerative disorders, such as Alzheimer's disease, needs to be considered.

The relatively small number of subjects and the lack of a control task condition represent further potential limitations of the present study. Furthermore, the placement of the reference electrode in a cephalic region (either anode or cathode) with an equally sized active electrode can induce reference-specific effects (anodal/ cathodal) in parallel to the cathodal/anodal effects of the active electrode. However, the present settings were used in well-established tDCS protocols to modulate the MFC (Adenzato et al., 2017; Bellaiche, Asthana, Ehlis, Polak, \& Herrmann, 2013). Another limitation in terms of the stimulation protocol is that we applied a single-session online tDCS design. This type of protocol focuses on short-term improvements in performance induced by a single session of stimulation, typically delivered online during the task. The main limitations are the lack of control conditions over different cortical areas and the lack of systematic monitoring of the duration of the effects.

The use of repeated tDCS sessions could be used to investigate the long-term effects of stimulation, which are particularly interesting in neurodegenerative patients, and the generalization of the tDCS effect on other cognitive abilities. Hence, further studies are needed in order to conclusively demonstrate the potential for the induction of long-term neuromodulatory effects using brain stimulation. In light of the present findings, the same protocol might be applied to bvFTD patients to elucidate long-term improvements in ToM performance.

Finally, it is important to note that studies that have investigated the neural substrates of social cognition in bvFTD have found the involvement of a broader brain network, including the frontoinsular regions (Dermody et al., 2016) and cerebellum, as well as the lateral temporal and occipitoparietal cortices (Synn et al., 2018). These findings suggest that the loss of social cognitive abilities in bvFTD could not be associated with the deterioration of a single brain region but reflect the decline of a distributed brain network that appears crucial for monitoring and processing social information. Similarly, the loss of ToM abilities could be linked to the deterioration of a distributed network, including the MFC. Thus, the stimulation of the MFC might increase the overall integrity of the functional ToM network. The application of network-based imaging methods should then be taken into consideration for future studies on ToM abilities in bvFTD, focusing on the divergent impact this disorder has on different brain areas and networks (Zhou \& Seeley, 2014).

\section{Conclusions}

Despite these limitations, our findings suggest that anodal tDCS applied over the MFC could be useful for enhancing a specific ToM ability (i.e., communicative intentions processing) and suggest that noninvasive brain stimulation could be employed to attempt to reduce the ToM deficits observed in bvFTD patients. In the present study, we used tDCS, which is a safe, noninvasive, and easily applied brain stimulation technique in which electrical current is directly applied to the head to generate an electrical field that modulates neuronal activity (Dayan, Censor, Buch, Sandrini, \& Cohen, 2013; Lefaucheur et al., 2017). It remains to be clarified whether the therapeutic effects of tDCS are clinically meaningful and how tDCS can be optimally performed in a therapeutic setting.

In this sense, the present study provides a significant contribution to the emergent domain of investigation that uses tDCS to improve social cognition (Santiesteban et al., 2012) and to treat neuropsychiatric disorders (Brunoni et al., 2012; Demirtas-Tatlidede, Vahabzadeh-Hagh, \& Pascual-Leone, 2013). Our findings fit well with these considerations and could potentially contribute to the development of an effective, noninvasive brain stimulation treatment for ToM impairments in patients with bvFTD. Further studies using multiple 
tDCS sessions and exploring the long-term effects of this approach are warranted.

Acknowledgements We wish to thank all the patients and caregivers who volunteered their time to participate in this study.

Mauro Adenzato was supported by the University of Turin (Ricerca scientifica finanziata dall'Università “Cognizione sociale e attaccamento in popolazioni cliniche e non cliniche"). Ivan Enrici was supported by University of Turin grants (Ricerca scientifica finanziata dall'Università "Linea Generale" and "Linea Giovani").

\section{Compliance with ethical standards}

Conflict of interest None

\section{References}

Adenzato, M., Brambilla, M., Manenti, R., De Lucia, L., Trojano, L., Garofalo, S., ... Cotelli, M. (2017). Gender differences in cognitive Theory of Mind revealed by transcranial direct current stimulation on medial prefrontal cortex. Scientific Reports, 7, 41219. doi:https:// doi.org/10.1038/srep41219

Adenzato, M., Cavallo, M., \& Enrici, I. (2010). Theory of mind ability in the behavioural variant of frontotemporal dementia: An analysis of the neural, cognitive, and social levels. Neuropsychologia, 48(1), 2 12. doi:https://doi.org/10.1016/j.neuropsychologia.2009.08.001

Adenzato, M., \& Garbarini, F. (2006). The As If in cognitive science, neuroscience and anthropology: A journey among robots, blacksmiths, and neurons. Theory \& Psychology, 16(6):747-759. https://doi.org/10.1177/0959354306070515

Adenzato, M., \& Poletti, M. (2013). Theory of mind abilities in neurodegenerative diseases: An update and a call to introduce mentalizing tasks in standard neuropsychological assessments. Clinical Neuropsychiatry, 10(5), 226-234.

Agarwal, S. M., Rajur, S., Bose, A., Shenoy, S., Miriyala, S., Shivakumar, V., ... Venkatasubramanian, G. (2016). Use of transcranial direct current stimulation (tDCS) in a woman with behavioral variant fronto-temporal dementia. Asian Journal of Psychiatry, 21, 31-32. doi:https://doi.org/10.1016/j.ajp.2016.02.007

Baez, S., Pinasco, C., Roca, M., Ferrari, J., Couto, B., Garcia-Cordero, I., ... Torralva, T. (2017). Brain structural correlates of executive and social cognition profiles in behavioral variant frontotemporal dementia and elderly bipolar disorder. Neuropsychologia. doi:https:// doi.org/10.1016/j.neuropsychologia.2017.02.012

Bara, B. G., Ciaramidaro, A., Walter, H., \& Adenzato, M. (2011). Intentional minds: A philosophical analysis of intention tested through fMRI experiments involving people with schizophrenia, people with autism, and healthy individuals. Frontiers in Human Neuroscience, 5, 7. doi:https://doi.org/10.3389/fnhum.2011.00007

Bara, B. G., Enrici, I., \& Adenzato, M. (2016). At the core of pragmatics: The neural substrates of communicative intentions. In S. L. Hickok \& S. L. Small (Eds.), Neurobiology of language. New York: Elsevier.

Bellaiche, L., Asthana, M., Ehlis, A. C., Polak, T., \& Herrmann, M. J. (2013). The modulation of error processing in the medial frontal cortex by transcranial direct current stimulation. Neuroscience Journal, 2013, 187692. doi:https://doi.org/10.1155/2013/187692

Bertoux, M., \& Hornberger, M. (2015). 'Try to see it my way': Which theory of mind tests best distinguish bvFTD and AD? Journal of Neurology, Neurosurgery \& Psychiatry, 86(7), 706. doi:https://doi. org/10.1136/jnnp-2015-310324
Borroni, B., Cosseddu, M., Pilotto, A., Premi, E., Archetti, S., Gasparotti, R., ... Padovani, A. (2015). Early stage of behavioral variant frontotemporal dementia: Clinical and neuroimaging correlates. Neurobiology of Aging, 36(11), 3108-3115. doi:https://doi.org/10. 1016/j.neurobiolaging.2015.07.019

Borroni, B., Grassi, M., Agosti, C., Paghera, B., Alberici, A., Di Luca, M., ... Padovani, A. (2007). Latent profile analysis in frontotemporal lobar degeneration and related disorders: Clinical presentation and SPECT functional correlates. BMC Neurology, 7, 9. doi:https://doi.org/10.1186/1471-2377-7-9

Boxer, A. L., \& Boeve, B. F. (2007). Frontotemporal dementia treatment: Current symptomatic therapies and implications of recent genetic, biochemical, and neuroimaging studies. Alzheimer Disease \& Associated Disorders, 21(4), S79-S87. doi:https://doi.org/10.1097/ WAD.0b013e31815c345e

Brioschi Guevara, A., Knutson, K. M., Wassermann, E. M., Pulaski, S., Grafman, J., \& Krueger, F. (2015). Theory of mind impairment in patients with behavioural variant fronto-temporal dementia (bvFTD) increases caregiver burden. Age and Ageing, 44(5), 891895. doi:https://doi.org/10.1093/ageing/afv059

Brune, M., \& Brune-Cohrs, U. (2006). Theory of mind-Evolution, ontogeny, brain mechanisms and psychopathology. Neuroscience \& Biobehavioral Reviews, 30(4), 437-455. doi:https://doi.org/10. 1016/j.neubiorev.2005.08.001

Brunoni, A. R., Nitsche, M. A., Bolognini, N., Bikson, M., Wagner, T., Merabet, L., ... Fregni, F. (2012). Clinical research with transcranial direct current stimulation (tDCS): Challenges and future directions. Brain Stimulation, 5(3), 175-195. doi:https://doi.org/10.1016/j.brs. 2011.03.002

Buhour, M. S., Doidy, F., Laisney, M., Pitel, A. L., de La Sayette, V., Viader, F., ... Desgranges, B. (2017). Pathophysiology of the behavioral variant of frontotemporal lobar degeneration: A study combining MRI and FDG-PET. Brain Imaging \& Behavior, 11(1), 240252. doi:https://doi.org/10.1007/s11682-016-9521-x

Carrington, S. J., \& Bailey, A. J. (2009). Are there theory of mind regions in the brain? A review of the neuroimaging literature. Human Brain Mapping, 30(8), 2313-2335. doi:https://doi.org/10.1002/hbm. 20671

Cavallo, M., Enrici, I., \& Adenzato, M. (2011). The comprehension of social situations in a small group of patients with frontotemporal dementia and Alzheimer's disease. Acta Neuropsychologica, 9, $167-176$.

Ciaramidaro, A., Adenzato, M., Enrici, I., Erk, S., Pia, L., Bara, B. G., ... Walter, H. (2007). The intentional network: How the brain reads varieties of intentions. Neuropsychologia, 45(13), 3105-3113. doi: https://doi.org/10.1016/j.neuropsychologia.2007.05.011

Conson, M., Errico, D., Mazzarella, E., Giordano, M., Grossi, D., \& Trojano, L. (2015). Transcranial electrical stimulation over dorsolateral prefrontal cortex modulates processing of social cognitive and affective information. PLOS ONE, 10(5), e0126448. doi:https://doi. org/10.1371/journal.pone. 0126448

Cotelli, M., Manenti, R., Paternico, D., Cosseddu, M., Brambilla, M., Petesi, M., ... Borroni, B. (2016). Grey matter density predicts the improvement of naming abilities after tDCS intervention in agrammatic variant of primary progressive aphasia. Brain Topogr, 29(5), 738-751. doi:https://doi.org/10.1007/s10548-016-0494-2

Cotelli, M., Manenti, R., Petesi, M., Brambilla, M., Cosseddu, M., Zanetti, O., ... Borroni, B. (2014). Treatment of primary progressive aphasias by transcranial direct current stimulation combined with language training. Journal of Alzheimer's Disease, 39(4), 799-808. doi:https://doi.org/10.3233/JAD-131427

Dayan, E., Censor, N., Buch, E. R., Sandrini, M., \& Cohen, L. G. (2013). Noninvasive brain stimulation: from physiology to network dynamics and back. Nat Neurosci, 16(7), 838-844. doi:https://doi.org/10. 1038/nn.3422 
Demirtas-Tatlidede, A., Vahabzadeh-Hagh, A. M., \& Pascual-Leone, A. (2013). Can noninvasive brain stimulation enhance cognition in neuropsychiatric disorders? Neuropharmacology, 64, 566-578. doi:https://doi.org/10.1016/j.neuropharm.2012.06.020

Dermody, N., Wong, S., Ahmed, R., Piguet, O., Hodges, J. R., \& Irish, M. (2016). Uncovering the neural bases of cognitive and affective empathy deficits in Alzheimer's disease and the behavioral-variant of frontotemporal dementia. Journal of Alzheimer's Disease, 53(3), 801-816. doi:https://doi.org/10.3233/JAD-160175

Di Tella, M., Castelli, L., Colonna, F., Fusaro, E., Torta, R., Ardito, R. B., ... Adenzato, M. (2015). Theory of Mind and emotional functioning in fibromyalgia syndrome: An investigation of the relationship between social cognition and executive function. PLOS ONE, 10(1), e0116542. doi:https://doi.org/10.1371/journal.pone.0116542

Enrici, I., Adenzato, M., Ardito, R. B., Mitkova, A., Cavallo, M., Zibetti, M., ... Castelli, L. (2015). Emotion processing in Parkinson's disease: A three-level study on recognition, representation, and regulation. PLOS ONE, 10(6), e0131470. doi:https://doi.org/10.1371/ journal.pone. 0131470

Enrici, I., Adenzato, M., Cappa, S., Bara, B. G., \& Tettamanti, M. (2011). Intention processing in communication: A common brain network for language and gestures. Journal of Cognitive Neuroscience, 23(9), 2415-2431. doi:https://doi.org/10.1162/jocn.2010.21594

Eslinger, P. J., Moore, P., Troiani, V., Antani, S., Cross, K., Kwok, S., \& Grossman, M. (2007). Oops! Resolving social dilemmas in frontotemporal dementia. Journal of Neurology, Neurosurgery \& Psychiatry, 78(5), 457-460. doi:https://doi.org/10.1136/jnnp.2006. 098228

Faul, F., Erdfelder, E., Lang, A. G., \& Buchner, A. (2007). G*Power 3: A flexible statistical power analysis program for the social, behavioral, and biomedical sciences. Behavior Research Methods, 39(2), 175191.

Fumagalli, M., Vergari, M., Pasqualetti, P., Marceglia, S., Mameli, F., Ferrucci, R., ... Priori, A. (2010). Brain switches utilitarian behavior: Does gender make the difference? PLOS ONE, 5(1), e8865. doi: https://doi.org/10.1371/journal.pone.0008865

Gervits, F., Ash, S., Coslett, H. B., Rascovsky, K., Grossman, M., \& Hamilton, R. (2016). Transcranial direct current stimulation for the treatment of primary progressive aphasia: An open-label pilot study. Brain and Language, 162, 35-41. doi:https://doi.org/10.1016/j. band1.2016.05.007

Gregory, C., Lough, S., Stone, V., Erzinclioglu, S., Martin, L., BaronCohen, S., et al. (2002). Theory of mind in patients with frontal variant frontotemporal dementia and Alzheimer's disease: Theoretical and practical implications. Brain, 125(Pt. 4), 752-764.

Henry, J. D., Phillips, L. H., \& von Hippel, C. (2014). A meta-analytic review of theory of mind difficulties in behavioural-variant frontotemporal dementia. Neuropsychologia, 56, 53-62. doi: https://doi.org/10.1016/j.neuropsychologia.2013.12.024

Herwig, U., Satrapi, P., \& Schonfeldt-Lecuona, C. (2003). Using the international 10-20 EEG system for positioning of transcranial magnetic stimulation. Brain Topography, 16(2), 95-99.

Ibanez, A., \& Manes, F. (2012). Contextual social cognition and the behavioral variant of frontotemporal dementia. Neurology, 78(17), 1354-1362. doi:https://doi.org/10.1212/WNL.0b013e3182518375

Irish, M., Hodges, J. R., \& Piguet, O. (2014). Right anterior temporal lobe dysfunction underlies theory of mind impairments in semantic dementia. Brain, 137(Pt. 4), 1241-1253. doi:https://doi.org/10.1093/ brain/awu003

Kampe, K. K., Frith, C. D., \& Frith, U. (2003). "Hey John": Signals conveying communicative intention toward the self activate brain regions associated with "mentalizing," regardless of modality. Journal of Neuroscience, 23(12), 5258-5263.

Kipps, C. M., \& Hodges, J. R. (2006). Theory of mind in frontotemporal dementia. Social Neuroscience, 1(3/4), 235-244. doi:https://doi.org/ $10.1080 / 17470910600989847$
Le Bouc, R., Lenfant, P., Delbeuck, X., Ravasi, L., Lebert, F., Semah, F., ... Pasquier, F. (2012). My belief or yours? Differential theory of mind deficits in frontotemporal dementia and Alzheimer's disease. Brain, 135(Pt. 10), 3026-3038. doi:https://doi.org/10.1093/brain/ aws 237

Lefaucheur, J. P., Antal, A., Ayache, S. S., Benninger, D. H., Brunelin, J., Cogiamanian, F., ... Paulus, W. (2017). Evidence-based guidelines on the therapeutic use of transcranial direct current stimulation (tDCS). Clinical Neurophysiology, 128(1), 56-92. doi:https://doi. org/10.1016/j.clinph.2016.10.087

Lezak, M., Howieson, D., \& Loring, D. (2012). Neuropsychological assessment (5th ed.). Oxford: Oxford University Press.

Lough, S., Gregory, C., \& Hodges, J. R. (2001). Dissociation of social cognition and executive function in frontal variant frontotemporal dementia. Neurocase, 7(2), 123-130. doi:https://doi.org/10.1093/ neucas/7.2.123

Lough, S., \& Hodges, J. R. (2002). Measuring and modifying abnormal social cognition in frontal variant frontotemporal dementia. Journal of Psychosomatic Research, 53(2), 639-646.

Lough, S., Kipps, C. M., Treise, C., Watson, P., Blair, J. R., \& Hodges, J. R. (2006). Social reasoning, emotion and empathy in frontotemporal dementia. Neuropsychologia, 44(6), 950-958. doi:https://doi.org/ 10.1016/j.neuropsychologia.2005.08.009

Mai, X., Zhang, W., Hu, X., Zhen, Z., Xu, Z., Zhang, J., ... Liu, C. (2016). Using tDCS to Explore the Role of the right temporoparietal junction in theory of mind and cognitive empathy. Frontiers in Psychology, 7, 380. doi:https://doi.org/10.3389/fpsyg. 2016.00380

Martin, A. K., Dzafic, I., Ramdave, S., \& Meinzer, M. (2017). Causal evidence for task-specific involvement of the dorsomedial prefrontal cortex in human social cognition. Social Cognitive and Affective Neuroscience, 12(8), 1209-1218. doi:https://doi.org/10.1093/scan/ nsx063

Martin, A. K., Huang, J., Hunold, A., \& Meinzer, M. (2017). Sex mediates the effects of high-definition transcranial direct current stimulation on "mind-reading". Neuroscience, 366, 84-94. doi:https://doi. org/10.1016/j.neuroscience.2017.10.005

Martins, A. R., Fregni, F., Simis, M., \& Almeida, J. (2017). Neuromodulation as a cognitive enhancement strategy in healthy older adults: promises and pitfalls. Aging, Neuropsychology, and Cognition, 24(2), 158-185. doi:https://doi.org/10.1080/13825585. 2016.1176986

Nitsche, M. A., Cohen, L. G., Wassermann, E. M., Priori, A., Lang, N., Antal, A., ... Pascual-Leone, A. (2008). Transcranial direct current stimulation: State of the art 2008. Brain Stimul, 1(3), 206-223.

Nitsche, M. A., Doemkes, S., Karakose, T., Antal, A., Liebetanz, D., Lang, N., et al. (2007). Shaping the effects of transcranial direct current stimulation of the human motor cortex. Journal of Neurophysiology, 97(4), 3109-3117. doi:https://doi.org/10.1152/jn. 01312.2006

Nitsche, M. A., Liebetanz, D., Lang, N., Antal, A., Tergau, F., \& Paulus, W. (2003). Safety criteria for transcranial direct current stimulation (tDCS) in humans. Clinical Neurophysiology, 114(11), 2220-2222; author reply 2222-2223.

Nitsche, M. A., \& Paulus, W. (2000). Excitability changes induced in the human motor cortex by weak transcranial direct current stimulation. Journal of Physiology, 527(Pt. 3), 633-639.

Pan, P. L., Song, W., Yang, J., Huang, R., Chen, K., Gong, Q. Y., ... Shang, H.-F. (2012). Gray matter atrophy in behavioral variant frontotemporal dementia: A meta-analysis of voxel-based morphometry studies. Dementia and Geriatric Cognitive Disorders, 33(2/3), 141-148. doi:https://doi.org/10.1159/000338176

Pardini, M., Emberti Gialloreti, L., Mascolo, M., Benassi, F., Abate, L., Guida, S., ... Cocito, L. (2013). Isolated theory of mind deficits and risk for frontotemporal dementia: A longitudinal pilot study. Journal 
of Neurology, Neurosurgery \& Psychiatry, 84(7), 818-821. doi: https://doi.org/10.1136/jnnp-2012-303684

Pardini, M., Serrati, C., Guida, S., Mattei, C., Abate, L., Massucco, D., ... Gialloreti, E. (2015). Souvenaid reduces behavioral deficits and improves social cognition skills in frontotemporal dementia: A proofof-concept study. Neurodegenerative Diseases, 15(1), 58-62. doi: https://doi.org/10.1159/000369811

Paulus, W. (2011). Transcranial electrical stimulation (tES — tDCS; tACS; tRNS) methods. Neuropsychological Rehabilitation, 21(5), 602-617.

Pezzati, R., Molteni, V., Bani M., Settanta C., Di Maggio M. G., Villa I., ... Ardito R. B. (2014). Can Doll therapy preserve or promote attachment in people with cognitive, behavioral, and emotional problems? A pilot study in institutionalized patients with dementia. Frontiers in Psychology, 5:342. https://doi.org/10.3389/fpsyg. 2014.00342

Perry, R. J., \& Miller, B. L. (2001). Behavior and treatment in frontotemporal dementia. Neurology, 56(11, Suppl. 4), S46-S51.

Polania, R., Nitsche, M. A., \& Ruff, C. C. (2018). Studying and modifying brain function with non-invasive brain stimulation. Nature Neuroscience, 21(2), 174-187. doi:https://doi.org/10.1038/s41593017-0054-4

Poletti, M., Enrici, I., \& Adenzato, M. (2012). Cognitive and affective Theory of Mind in neurodegenerative diseases: Neuropsychological, neuroanatomical and neurochemical levels. Neuroscience \& Biobehavioral Reviews, 36(9), 2147-2164. doi: https://doi.org/10.1016/j.neubiorev.2012.07.004

Poreisz, C., Boros, K., Antal, A., \& Paulus, W. (2007). Safety aspects of transcranial direct current stimulation concerning healthy subjects and patients. Brain Research Bulletin, 72(4/6), 208-214.

Premi, E., Grassi, M., van Swieten, J., Galimberti, D., Graff, C., Masellis, M., ... GENFI Consortium members. (2017). Cognitive reserve and TMEM106B genotype modulate brain damage in presymptomatic frontotemporal dementia: A GENFI study. Brain, 140(6), 17841791. doi:https://doi.org/10.1093/brain/awx103

Rascovsky, K., Hodges, J. R., Knopman, D., Mendez, M. F., Kramer, J. H., Neuhaus, J., ... Miller, D. (2011). Sensitivity of revised diagnostic criteria for the behavioural variant of frontotemporal dementia. Brain, 134(Pt. 9), 2456-2477. doi:https://doi.org/10.1093/brain/ awr179

Roncero, C., Kniefel, H., Service, E., Thiel, A., Probst, S., \& Chertkow, H. (2017). Inferior parietal transcranial direct current stimulation with training improves cognition in anomic Alzheimer's disease and frontotemporal dementia. Alzheimers Dement (NY), 3(2), 247253. doi:https://doi.org/10.1016/j.trci.2017.03.003

Salmon, E., Garraux, G., Delbeuck, X., Collette, F., Kalbe, E., Zuendorf, G., ... Herholz, K. (2003). Predominant ventromedial frontopolar metabolic impairment in frontotemporal dementia. NeuroImage, 20(1), 435-440.

Santiesteban, I., Banissy, M. J., Catmur, C., \& Bird, G. (2012). Enhancing social ability by stimulating right temporoparietal junction. Current Biology, 22(23), 2274-2277. doi:https://doi.org/10.1016/j.cub. 2012.10.018
Schroeter, M. L., Raczka, K., Neumann, J., \& von Cramon, D. Y. (2008). Neural networks in frontotemporal dementia-A meta-analysis. Neurobiology of Aging, 29(3), 418-426. doi:https://doi.org/10. 1016/j.neurobiolaging.2006.10.023

Snowden, J. S., Gibbons, Z. C., Blackshaw, A., Doubleday, E., Thompson, J., Craufurd, D., ... Neary, D. (2003). Social cognition in frontotemporal dementia and Huntington's disease. Neuropsychologia, 41(6), 688-701.

Synn, A., Mothakunnel, A., Kumfor, F., Chen, Y., Piguet, O., Hodges, J. R., ... Irish, M. (2018). Mental states in moving shapes: Distinct cortical and subcortical contributions to theory of mind impairments in dementia. Journal of Alzheimer's Disease, 61(2), 521-535. doi: https://doi.org/10.3233/jad-170809

Tettamanti, M., Vaghi, M. M., Bara, B. G., Cappa, S. F., Enrici, I., \& Adenzato, M. (2017). Effective connectivity gateways to the Theory of Mind network in processing communicative intention. Neuroimage, 155, 169-176. doi:https://doi.org/10.1016/j. neuroimage.2017.04.050

Torralva, T., Kipps, C. M., Hodges, J. R., Clark, L., Bekinschtein, T., Roca, M., ... Manes, F. (2007). The relationship between affective decision-making and theory of mind in the frontal variant of frontotemporal dementia. Neuropsychologia, 45(2), 342-349. doi:https:// doi.org/10.1016/j.neuropsychologia.2006.05.031

Vallar, G., \& Bolognini, N. (2011). Behavioural facilitation following brain stimulation: Implications for neurorehabilitation. Neuropsychological Rehabilitation, 21(5), 618-649. doi:https:// doi.org/10.1080/09602011.2011.574050

Van Overwalle, F. (2009). Social cognition and the brain: A meta-analysis. Human Brain Mapping, 30(3), 829-858. doi:https://doi.org/10. $1002 / \mathrm{hbm} .20547$

Van Overwalle, F. (2011). A dissociation between social mentalizing and general reasoning. NeuroImage, 54(2), 1589-1599. doi:https://doi. org/10.1016/j.neuroimage.2010.09.043

Van Overwalle, F., \& Baetens, K. (2009). Understanding others' actions and goals by mirror and mentalizing systems: A meta-analysis. NeuroImage, 48(3), 564-584. doi:https://doi.org/10.1016/j. neuroimage.2009.06.009

Walter, H., Adenzato, M., Ciaramidaro, A., Enrici, I., Pia, L., \& Bara, B. G. (2004). Understanding intentions in social interaction: The role of the anterior paracingulate cortex. Journal of Cognitive Neuroscience, 16(10), 1854-1863. doi:https://doi.org/10.1162/ 0898929042947838

Walter, H., Ciaramidaro, A., Adenzato, M., Vasic, N., Ardito, R. B., Erk, S., ... Bara, B. G. (2009). Dysfunction of the social brain in schizophrenia is modulated by intention type: An fMRI study. Social Cognitive and Affective Neuroscience, 4(2), 166-176. doi:https:// doi.org/10.1093/scan/nsn047

Zhou, J., \& Seeley, W. W. (2014). Network dysfunction in Alzheimer's disease and frontotemporal dementia: Implications for psychiatry. Biological Psychiatry, 75(7), 565-573. doi:https://doi.org/10.1016/ j.biopsych.2014.01.020 